\title{
Designing 3D Menu Interfaces for Spatial Interaction in Virtual Environments
}

\author{
JoungHuem Kwon ${ }^{1}$, Jung-Yoon $\mathrm{Kim}^{2}$ and SangHun Nam ${ }^{3 *}$ \\ ${ }^{1}$ School of Digital Cultual and Contents, Youngsan University, \\ Bansong-ro 142, Heawonde-gu, Busan, South Korea \\ ${ }^{2}$ Graduate School of Game, Gachon University, \\ 1342 Seongnam Daero, Sujeong-Gu, Seongnam-Si, Gyeonggi-Do, South Korea \\ ${ }^{3}$ Dept. of Newmedia, Seoul Media Institute of Technology, \\ 99, Hwagok-ro 61-gil, Gangseo-gu, Seoul, South Korea \\ hjkwon@ysu.ac.kr,kjyoon79@gmail.com,*shnam@smit.ac.kr
}

\begin{abstract}
This paper proposes several concepts for spatial menu interfaces in immersive virtual environments. Virtual Reality $(V R)$ is a new interactive medium of immersive experience and VR user interfaces must be natural and seamless in $3 D$ environments. Designing new $V R$ interfaces is necessary because traditional desktop user interfaces can reduce the immersion experience and make VR cumbersome to use. This study includes comparing, contrasting, analyzing and evaluation the existing spatial user interface and $3 D$ menu interfaces to interact with users in a virtual environment. This study will be used to support the next phases of design and technical development.
\end{abstract}

Keywords: Virtual Reality, Spatial User Interface, Metaphor

\section{Introduction}

Virtual Reality (VR) is a new interactive medium of immersive experience that drives our brain to believe in the virtual world as if it were the actual one. To achieve this, user interfaces must be natural and seamless to avoid the dilution of such experiences. In VR, it is important to imitate sensations through the accompanying hardware, such that the user can get a sense of the real world even though he is in the virtual world. The VR system is also very important to provide some cues that the user exists in the threedimensional space. Like other interactive computing systems, VR interfaces must support the selection, positioning, and rotation of virtual objects, drawing lines, numeric values, and text input assignments. WIMP (windows, icons, menus, and pointing) does not work well with VR systems, just like traditional desktop UI metaphors. Not only is interaction required with six degrees of freedom (6DOF) rather than $2 \mathrm{DOF}$, but the use of traditional devices (like a mouse and keyboard) can reduce the immersion experience and make VR cumbersome to use. Therefore, designing a new interface for a VR system is necessary.

There are many references to the importance of interface design. It is desirable to have an interface design that allows users to effectively access the system in a way that meets their personal needs, with an efficiency that speeds up data entry and reduces errors. This is the final goal of the interface design, which many studies have focused on.

Recently, various new UI paradigms have been proposed along with the emergence of VR contents. One example is to allow the users to highlight and select menus in a virtually created environment using head movements and hand gestures. Another example

Received (October 5, 2017), Review Result (November 29, 2017), Accepted (December 4, 2017)

* Corresponding Author 
is the use of a handheld controller to move a virtual space, manipulate a virtual object, and interact with the surrounding virtual environment. The menu selection is made by clicking a few buttons, very similarly to a conventional joystick controls. The interface presented so far can adequately provide the user with a spatial experience, but the menu is no different from the existing WIMP method in operation. Pointing and clicking in 3D space are difficult tasks for users who are previously familiar with $2 \mathrm{D}$ systems. In particular, the method for moving the cursor by rotating the head has in the limitation that it requires not only long practice for the user to get familiar with it, but also limits the immersion feeling to the space.

The motivation of this study lies in the proposal of a new 3D menu design that considers spatial interaction in virtual environments. Firstly, this research has conducted a study for comparing, contrasting, analyzing and evaluating the existing spatial user interfaces in a virtual environment in order to help the authors in learning the UI techniques, which include menus and extra information about objects and UI elements in a space. Secondly, we present several design concepts for 3D spatial user interfaces in virtual environments. Also, the design process for giving the UIs an acceptable form is presented to users. This is an ongoing project, currently at an estimated midway point of the whole development cycle, so it is also partly treated as an experiment. This study will be used to support the next phase: design and technical development.

\section{Related Works}

Metaphor can be a powerful tool for designers in both the process of designing and within the products themselves. In designing a graphical user interface, metaphor is frequently used to help users understand how to use the system. Here we introduce several cases in the concept of graphical user interfaces that have been proposed by various organizations. These cases show that the most common metaphors are Desktop, Room, Card Deck, and Art Gallery, all of which are instrumental in delivering a 3D experience to a user (See Figure 1).

One actual example of delivering a user a spatial experience is a home interface that has been implemented as a metaphorical living room in both SteamVR and Oculus. As in the real living room, the virtual home interface lets us go inside, see bookshelves, pick up a multimedia corresponding to an application execution, and grasp and throw virtual properties. In addition, built-in social functions make it easier than ever to connect among friends or other players. Another function is the allowance to invite friends to join us in our home space. Voice chat with other players, interaction with tools and props, and exploration of different VR environments with others is also possible.

To build a user's identity, the avatar creator has also been implemented. A user can choose among several avatar's appearances, such as head, accessories and clothes through the selection of specific icons. The metaphorical use of the living room interface seems to be a perfect UI to deliver various contents with the spatial experience, however, it remains still limited to a menu selection. Once a user clicks a controller's button, the UI shows up a $2 \mathrm{D}$ screen that allows us to change options of the system menu. The virtual keyboard also does imply a precise interaction for managing an environment of 6 DOF. 


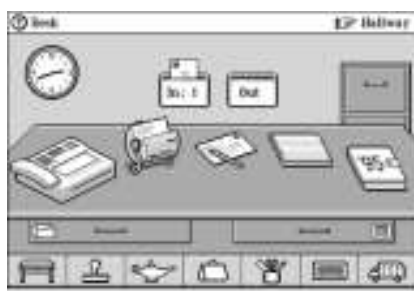

Desktop

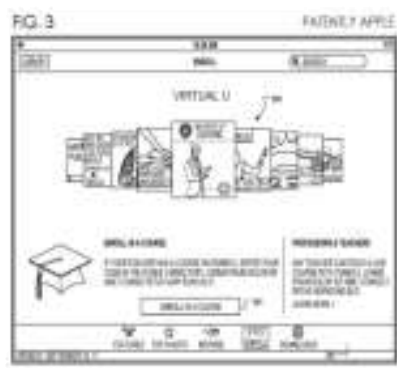

Art gallery

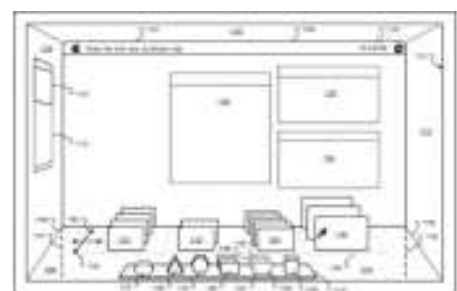

Room

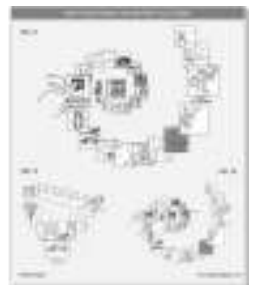

Card

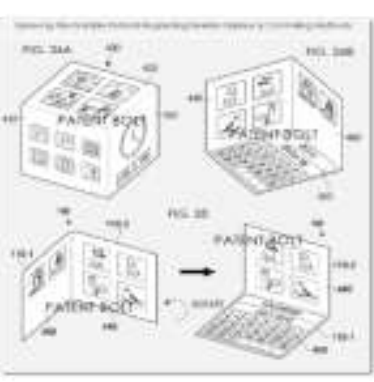

Cube

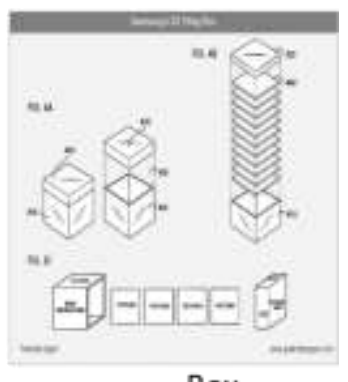

Box

\section{Figure 1. Implied Metaphors for Graphic User Interface Design}

The most common approach for selecting a technique in 3D menus is the type of raycast. Users can directly point to an object for selecting, with the use of a virtual ray, as it is the actual "laser pointer" device. This technique only requires the user to control 2 DOF, however, it shows difficulty to select especially layered objects [1]. Another method is given by the use of the virtual fingertip as a tool for menu selection. It uses a one-to-one mapping method that is similar to the real world in a very intuitive way. The limitation of this method is that the user feels much difficulty to perceive depth in a virtual environment, moreover, the user can only select menu within the hand reach [2].

Recently, Achibet $t$ and his colleagues [3] proposed an "elastic-arm" to overcome the limitation given by the use of virtual fingertip. The menu selection can be performed by the stretching of virtual arms. Given that the elastic-arm has a hybrid ray cast and fingertip technique this method does not require large user movements to select a menu, even at a long distance. Also, it has a valuable menu selection, even under certain difficulty in depth sensing in 3D environments. The weakness of this method lies on the fact that users find very difficult to learn the menu selection due to the non-linear mapping between the physical and the virtual position of the hand.

One more example is the scaled world grab technique [4], which is similar to the electric-arm method, but differentiated by having shown being better in occluded and small object selection. The basic principle is that the selected object is decomposed and enlarged so that it is reconstructed to make the selection easier for the user, who can partially manipulate the decomposed object and select the overlapped object. The main disadvantage is that it is difficult to memorize the shape of the original image due to the screen reconstruction. As shown in the actual experiment, the performance of selecting a specific object has improved, but it remains difficult the task of restoring it as a full object model. 
Table 1. Techniques for Selection in VR

\begin{tabular}{|c|c|c|c|c|}
\hline Techniques & Explanation & Pros. & Cons. & Example \\
\hline Ray-cast [1] & $\begin{array}{l}\text { It is used a } \\
\text { virtual light ray } \\
\text { to select objects }\end{array}$ & $\begin{array}{l}\text { It only } \\
\text { needs to } \\
\text { control } 2 \\
\text { dof. }\end{array}$ & $\begin{array}{l}\text { Difficulty on } \\
\text { selecting second } \\
\text { layered objects }\end{array}$ & 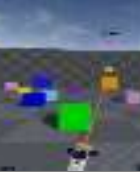 \\
\hline $\begin{array}{l}\text { Virtual hand } \\
\text { fingertip [2] }\end{array}$ & $\begin{array}{c}\text { It is used a } \\
\text { virtual fingertip } \\
\text { as a tool for } \\
\text { selecting } \\
\text { objects }\end{array}$ & $\begin{array}{l}\text { One to } \\
\text { one } \\
\text { mapping } \\
\text { (natural) }\end{array}$ & $\begin{array}{l}\text { Difficulty for } \\
\text { perceiving depth. } \\
\text { Only allows } \\
\text { selection of } \\
\text { objects at a hand } \\
\text { reach } \\
\end{array}$ & \\
\hline $\begin{array}{c}\text { Elastic-arm } \\
\text { [3] }\end{array}$ & $\begin{array}{c}\text { The user } \\
\text { performs a } \\
\text { selection task } \\
\text { by stretching } \\
\text { his virtual arm }\end{array}$ & $\begin{array}{c}\text { It is easy } \\
\text { to reach } \\
\text { distant } \\
\text { objects }\end{array}$ & $\begin{array}{l}\text { Non-linear } \\
\text { mapping between } \\
\text { physical and } \\
\text { virtual hand } \\
\text { position }\end{array}$ & \\
\hline $\begin{array}{c}\text { Scaled- } \\
\text { world grab } \\
{[4]}\end{array}$ & $\begin{array}{c}\text { The user } \\
\text { performs a } \\
\text { selection task } \\
\text { by stretching } \\
\text { his virtual arm }\end{array}$ & $\begin{array}{l}\text { Easy to } \\
\text { reach } \\
\text { distant } \\
\text { objects }\end{array}$ & $\begin{array}{l}\text { It is difficult to } \\
\text { notice a change in } \\
\text { the image }\end{array}$ & \\
\hline
\end{tabular}

Some research on virtual environment system control has been carried out. System control is related to virtual information that may appear in some way, and associates an effect parameter to changes over the system state or mode. This allows a user to interactively control an effect parameter, i.e. launching the application, changing system settings, and opening a media file. The issuing of a command always includes the selection of an element from a set to which belongs, thus, some similarities may exist between system control and menu selection.

The most common form in the system menu is given by the use of a graphic card metaphor. This is a method for selecting a menu by arranging a plurality of cards in a three-dimensional space. On the top of the card, there are icons and graphic symbols which are often used for on/off commands [5]. The virtual keyboard is also related to one of the common methods to insert some text or for changing parameters [5]. Both of them are aimed to provide the user with "familiarity" by applying the information input method mainly used in the two-dimensional interaction as a 3-dimensional space, but many researchers have pointed out the limitation of the depth awareness, which is the characteristic of the 3-dimensional space.

Table 2. System Menu in VR

\begin{tabular}{ccccc}
\hline Techniques & Explanation & Pros. & Cons. & Example \\
$\begin{array}{c}\text { Graphic } \\
\text { Card [5] }\end{array}$ & $\begin{array}{c}\text { Using icons or } \\
\text { symbol to } \\
\text { on/off mode }\end{array}$ & $\begin{array}{c}\text { Common } \\
\text { method }\end{array}$ & $\begin{array}{c}\text { Only On-Off } \\
\text { mode available }\end{array}$ \\
$\begin{array}{c}\text { Virtual } \\
\text { keyboard } \\
\text { [6] }\end{array}$ & $\begin{array}{c}\text { Using virtual } \\
\text { keyboard to } \\
\text { type text }\end{array}$ & Familiar & $\begin{array}{c}\text { Difficult to } \\
\text { perceive depth } \\
\text { to click keys }\end{array}$ \\
$\begin{array}{c}\text { Touch and } \\
\text { slide bar } \\
\text { [7] }\end{array}$ & $\begin{array}{c}\text { Using slide to } \\
\text { change } \\
\text { parameters }\end{array}$ & Familiar & $\begin{array}{c}\text { Same as } \\
\text { desktop } \\
\text { interaction }\end{array}$ &
\end{tabular}




\section{Design Process for Spatial UIs}

Through previous studies, we were able to identify several important issues. In the 3D space, the user may have difficulty in manipulating the object or touching the specific menu due to the lack of information about the virtual space depth. Although the ray cast method is partially adapted to overcome the limitation of depth perception, the change of the user's view is unavoidable given that overlap between the menus occurs while menu selection. In addition, when using a hand-based direct object manipulation metaphor, performance depends on the accuracy of the sensor. Due to these various limitations, it is necessary to propose a new type of interface in a three-dimensional space.

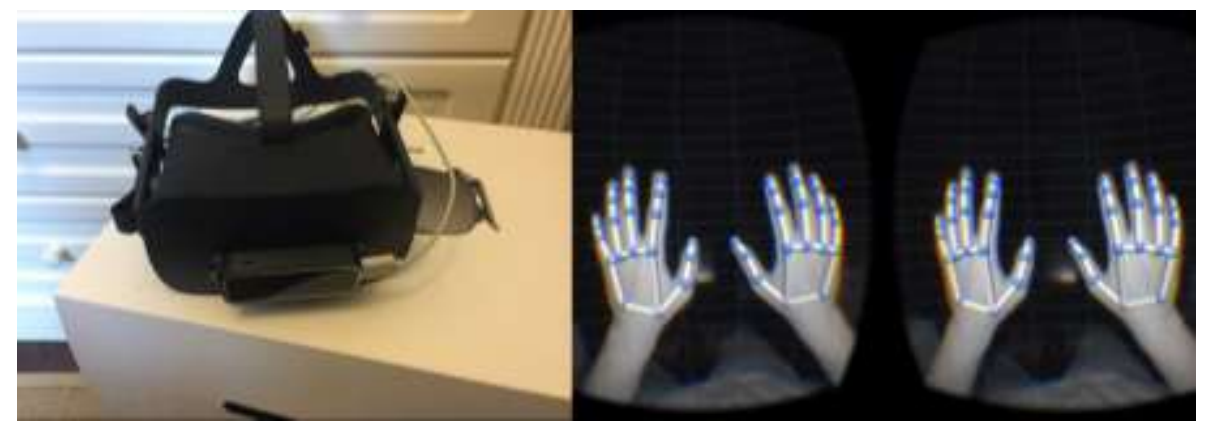

Figure 2. VR system with a Leapmotion Sensor

To construct the prototype of the proposed user interface and test its performance, we constructed the platform as follows: Oculus for HMD, i7 with GTX1080 for controlling the system and Leapmotion for obtaining the user's hand motion (mainly the rotation value). Leapmotion was set to the front of the HMD for configuration of an ego-centric view. Unity v5.5 software was used for prototyping the graphical menu interface. The whole system can be illustrated as shown in Figure 2.

\subsection{Prism}

Prism is an optical tool that refracts and disperses light. This has often been experienced by anyone involved in scientific practice, and it has recently been used to play culture. Using the principle of light refraction and straightness, descriptive of prism, the virtual bracelet worn on the wrist is the starting point of the menu. Submenus are unfolded and selected according to the direction to which the wrist rotates. It can be seen that the sensor that can measure the wrist rotation value in a very simple way, and is able to select and activate the main menu and sub menus. The prototype of the menu interface is shown in Figure 3. 


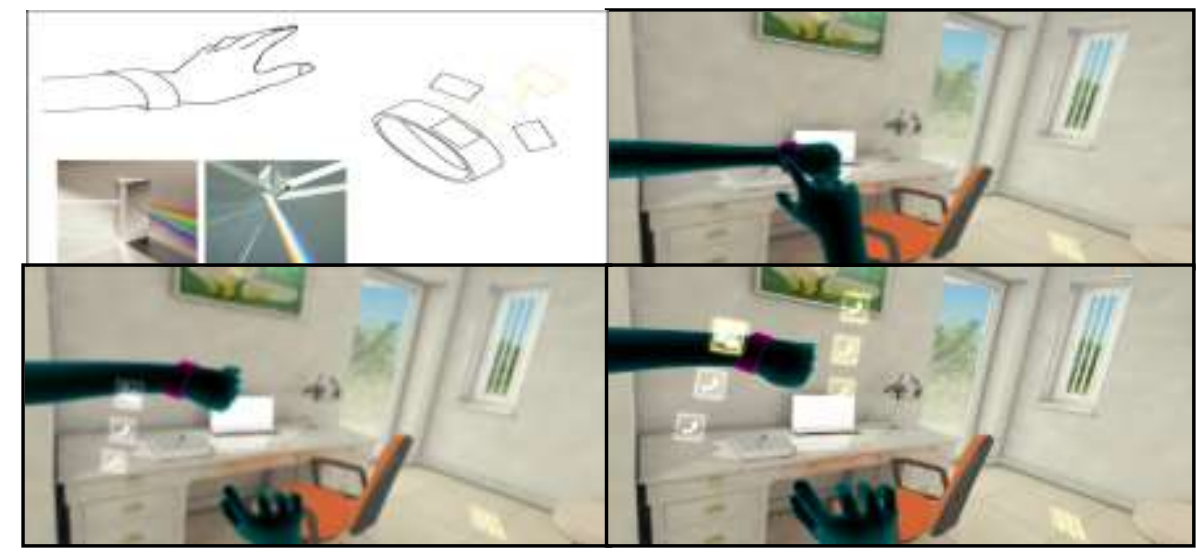

Figure 3. The concept of UI Menu for Spatial Interaction: Prism

\subsection{Jog Shuttle}

Using the jog shuttle metaphor, the user manipulates the virtual jog dial. The jog dial has a one-way rotation and pushing function. When a push occurs, the menu selection mode is opened and then the sub-menu can be selected by rotation. To expand the submenu, press again on the selected upper menu. The user can call, highlight, and select a menu with only a specific rotation and pressing action. It is the method that most automobiles use now and it is applied to easily select menus during operation, the prototype of menu interface can be illustrated in Figure 4.

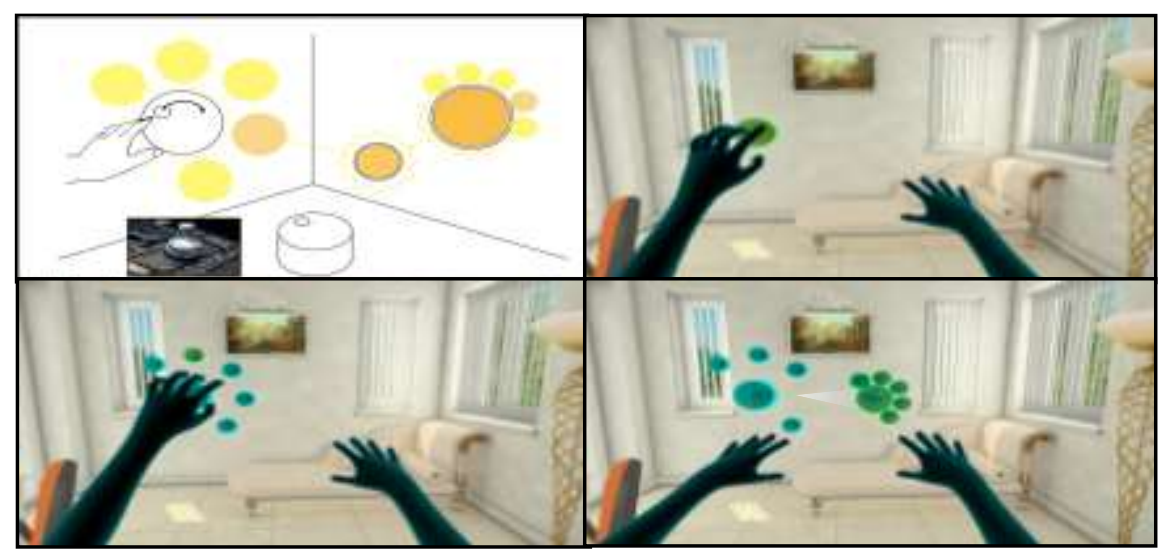

Figure 4. The concept of UI Menu for Spatial Interaction: Jog Shuttle

\section{Conclusion}

This paper was focused on the existing UIs for spatial interaction in immersive virtual environments. It proposes several concepts for spatial user interfaces as a consequence of related works. Although various attempts have been made to efficiently interact with users in virtual environments, such attempts have clearly demonstrated their limitations, hence the need of new ideas to improve them has been made evident. The proposed 3D Menu Interface has shown obvious drawbacks however, it is expected that several attempts lead to advancement on 3D menus. This project is currently ongoing and at an estimated midway point of the whole development cycle, so, it is also partly treated as an experiment. This study will be used to support the next phases of design and technical development. Further studies will be conducted to investigate how the proposed 3D menu is effective from the perspective of spatial experience. 


\section{Acknowledgments}

This research is supported by Ministry of Culture, Sports and Tourism(MCST) and Korea Creative Content Agency(KOCCA) in the Culture Technology(CT) Research \& Development Program 2017 (R2017030062_00000001)

\section{References}

[1] R.M.S. Clifford, N. M. B. Tuanquin and R. W. Lindeman, "Jedi ForceExtension: Telekinesis as a Virtual Reality interaction metaphor”, 3D User Interfaces (3DUI), 2017 IEEE Symposium on. IEEE, (2017).

[2] Y. Jang, "3d finger cape: Clicking action and position estimation under self-occlusions in egocentric viewpoint", IEEE transactions on visualization and computer graphics, vol. 21, no. 4, (2015), pp. 501510.

[3] M. Achibet, "Elastic-Arm: Human-scale passive haptic feedback for augmenting interaction and perception in virtual environments", Virtual Reality (VR), 2015 IEEE. IEEE, (2015).

[4] J. Lee, "Fast and Accurate 3D Selection using Proxy with Spatial Relationship for Immersive Virtual Environments", Proceedings of the 2016 Symposium on Spatial User Interaction. ACM, (2016).

[5] W. Scott, Greenwald, W. Corning and P. Maes. "Multi-User Framework for Collaboration and CoCreation in Virtual Reality", 12th International Conference on Computer Supported Collaborative Learning (CSCL), (2017).

[6] Unreal Engine Homepage, https://docs.unrealengine.com/latest/INT/Engine/Editor/VR/ GDC2017/, last accessed 2017/5/24.

[7] J.-H. Kwon, "Be closer as you are being there: HMD-based social interaction system", SIGGRAPH ASIA 2016 VR Showcase. ACM, (2016). 
International Journal of Grid and Distributed Computing Vol. 10, No. 12 (2017) 